\title{
An In-Home Synagis Program for RSV Prevention in High-Risk Infants
}

\begin{abstract}
OBJ ECTIVES: To evaluate and describe outcomes associated with a novel inhome administration program of respiratory syncytial virus (RSV) prophylaxis with palivizumab; to analyze health resource utilization and cost outcomes; and to assess parental satisfaction with in-home administration of prophylaxis for high-risk children.
\end{abstract}

DESIGN: Descriptive program evaluation.

SETTING: A national in-home RSV prophylaxis program for high-risk infants and children.

PARTICIPANTS: 396 infants and chitdren at risk for serious RSV infection.

\section{MAIN OUTCOME MEASURES:}

Incidence of RSV-related hospitalizations and emergency department (ED) visits, adverse events, program and hospitalization costs, compliance, and satisfaction.

RESULTS: Compared with published reports of office, outpatient, or clinicbased RSV prophylaxis with palivizumab, in-home prophylaxis demonstrated low incidence of total hospitalizations ( $11.4 \%$ versus $14.4 \%$ ) and RSV-related hospitalizations

( $2.3 \%$ versus $4.8 \%$ ). Less than $10 \%$ of patients required an ED visit and only $1 \%$ of those were related to RSV illness. Only one of the hospitalized infants required ICU admission. One- fourth of the patients experienced fever or ear infection; $43.2 \%$ developed cold symptoms. In-home palivizumab prophylaxis has the potential for saving $\$ 214$ per patient over drug administration and hospitalization costs. Parental satisfaction with the in-home program was $98.6 \%$, leading to high compliance; less than $2 \%$ of patients discontinued the program due to noncompliance.

CONCLUSIONS: In-home administration of palivizumab RSV prophylaxis can provide favorable outcomes, high parent satisfaction, and potential cost savings. Incidence of RSV-related hospitalizations among patients given prophylaxis at home was substantially lower than has been reported with outpatient or clinic-based prophylaxis. Compliance was excellent due to ease of administration of intramuscular palivizumab, convenience for families with multiple or premature infants or infants requiring cumbersome medical devices, and parents' desire to minimize the risk of exposure to RSV and other communicable diseases in clinics. Improved clinical outcomes observed with in-home prophylaxis may translate into substantial cost savings for health care purchasers.

\section{KEYWORDS: Home care,} Immunization, Synagis, Respiratory syncytial virus, Gentiva

J Managed Care Pharm 2001: 476-81

\section{Authors}

MARY WEBER, R.N., is National Director-Clinical Development, Gentiva Health Services; STEVEN REN O, R.Ph., is Manager, Clinical Pharmacy Practice, Gentiva Health Services; RODNEY HORNBAKE, M.D., is Senior Vice President and Chief Medical Officer, Gentiva Health Services, Melville, NY; and ALICIA SHILLINGTON, R.N., M.P.H., is Vice President, Clinical Research, EPIQ, Inc., Oakbrook Terrace, IL, and Adjunct Clinical Professor, University of Illinois at Chicago College of Pharmacy.

AUTHOR CORRESPONDENCE: Rodney Hornbake, MD, 3 Huntington Quadrangle, 2S M elville, NY 11747-7482; Tel: 631-501-7062; Fax: 913-8145475; E-mail: Rodney.H ornbake@gentiva.com.

Copyright@ 2001 Academy of M anaged Care Pharmacy, Inc. All rights reserved.

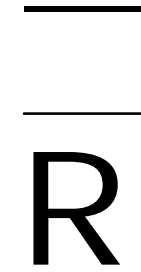

by Mary Weber, Steven Reno, Rodney Hornbake, and Alicia Shillington two years of age. ${ }^{1}$ It is transmitted through contact with contaminated air particles, secretions, or contaminated surfaces. ${ }^{1}$ RSV infections are particularly prevalent during the winter and early spring. Although they are usually mild and self-limiting, RSV infections are a major cause of morbidity and mortality in certain pediatric groups, including premature infants (less than 32 weeks gestation) and those with chronic lung disease (CLD), bronchopulmonary dysplasia (BPD), congenital heart disease, multiple congenital abnormalities, and certain immunod eficiencies. ${ }^{1,2}$ In the United States, more than 90,000 hospitalizations and 4,500 deaths occur each year as a result of RSV and its complications. ${ }^{2}$

Before 1998, the only effective means of passive immunization against RSV was respiratory syncytial virus immune globulin (RSV-IGIV, RespiGam, Massachusetts Public Health Biologic Laboratories, Boston, Massachusetts), a sterile human immunoglobulin derived from pooled adult human plasma. ${ }^{2}$ In 1998 the U.S. Food and Drug Administration (FDA) approved palivizumab (Synagis, Medimmune, Inc., Gaithersburg, Maryland), an RSV monoclonal antibody, for prophylaxis of RSV infections. ${ }^{2}$

Palivizumab acts as a specific antigenic site in RSV subtypes $A$ and $B$ to inhibit replication of RSV and directly neutralize the virus in nasal and tracheal aspirates. ${ }^{3}$ As a monoclonal antibody, palivizumab has a smaller volume than the RSV human immune globulin; therefore, it can be administered by intramuscular injection or intravenous infusion. ${ }^{3}$ In dose-finding studies, mean 30-day trough serum concentrations after each of five monthly intramuscular or intravenous injections of 15 $\mathrm{mg} / \mathrm{kg}$ in high-risk infants were consistently above $40 \mathrm{mcg} / \mathrm{mL}$, the concentration required to inhibit pulmonary RSV replication in animals. ${ }^{2-4}$

The effectiveness of palivizumab as RSV prophylaxis was assessed in a multi-center randomized double-blind placebocontrolled trial of more than 1,500 premature infants or infants with BPD who were treated in hospital outpatient clinics. ${ }^{2}$ When administered every 30 days for 5 months, palivizumab significantly reduced the incidence of RSV-related hospitalization, by $55 \%$ ( $4.8 \%$ versus $10.6 \%, p<0.00004)$, and its effectiveness remained statistically significant when adjusted for gender, presence of BPD, age or weight at study entry, and ges- 
An In-Home Synagis Program for RSV Prevention in High-Risk Infants

tational age $(p<0.014)$. Furthermore, the infants receiving palivizumab prophylaxis had a significantly shorter hospital length of stay (LOS), fewer hospital days on mechanical ventilation, fewer intensive care unit (ICU) admissions, and significantly shorter ICU LOS attributable to RSV $(p \varangle 0.026)$. Compliance with the five-injection schedule was $92 \%$; discontinuation due to palivizumab-related adverse events was rare $(0.3 \%) .^{2}$

Palivizumab was well tolerated in these clinical studies. ${ }^{2,4}$ O ne child with serious BPD treated in the intramuscular dosefinding study experienced fever, diarrhea, and exacerbation of respiratory symptoms after intramuscular injection of $5 \mathrm{mg} / \mathrm{kg}$; two others had mild and transient erythema at the intravenous injection site with the 5 and $15 \mathrm{mg} / \mathrm{kg}$ doses. ${ }^{4}$ In the comparative trial, the incidence of adverse events reported for the palivizumab and placebo-treated patients was similar ( $11 \%$ and $10 \%)$, with fever, irritability, and injection site reactions being the most common. ${ }^{2}$ Reactions at the intramuscular injection site occurred at $2.7 \%$ with palivizumab and $1.8 \%$ with placebo, and usually consisted of mild and transient erythema.

The literature describes only office and clinic-based palivizumab programs. Based on the safety and effectiveness of palivizumab prophylaxis demonstrated by these studies, and the ease of intramuscular administration of the monoclonal antibody, it seemed possible to extend RSV prophylaxis to a broader range of infants at high risk and facilitate RSV prophylaxis at home., ${ }^{2,4}$

As one of the largest providers of pediatric home care in the United States and Canada, Gentiva Health Services (Gentiva) cares for many infants and children who have experienced serious complications related to premature birth and chronic pulmonary disease and who are considered at high risk of serious RSV disease. Gentiva previously provided RSV-IGIV prophylaxis at home to infants at high risk of severe RSV illness. Parent preference indicated high satisfaction with the in-home program, especially the minimized exposure of high-risk infants to infectious disease compared to the clinic setting. Parents also noted diminished barriers related to transporting infants and requisite medical devices. Having followed the progress of the palivizumab clinical trials closely, Gentiva implemented a similar in-home program following the drug's approval by the FDA. In addition, Gentiva implemented a system of evaluation in order to determine the benefits of the program to children and payers.

Objectives

The primary objective of the in-home program for the 1999-2000 RSV season was to demonstrate the outcomes of inhome RSV prophylaxis with palivizumab. Secondary objectives were to investigate utilization and cost impact outcomes and assess parental satisfaction.

Program Design

The program was implemented in 16 states (Washington, Minnesota, Arkansas, Arizona, Florida, Iowa, Idaho, Kentucky,
Louisiana, Michigan, N ew Jersey, Oregan, South Dakota, Texas, Virginia, and Wisconsin) during the 1999-2000 RSV season. Staff were trained for home administration of palivizumab using a detailed written procedure and $35-\mathrm{mm}$ slides that covered RSV and palivizumab, a description of the in-home RSV prophylaxis program, patient eligibility criteria, nursing criteria, initial and ongoing patient assessments, and administration procedures. Only registered nurses (RNs) with a minimum of one year of neonatal or infant experience in an acute care setting and a passing score on the pediatric pre-employment test participated. They were also required to complete a pediatric orientation program and the palivizumab educational program.

Prospective patients were referred for the in-home program by discharge planners or social workers, neonatal intensive care units, pediatricians, pediatric pulmonologists, and medical insurance care managers.

Patient eligibility was assessed using the guidelines and recommendations for palivizumab prophylaxis published by the American Academy of Pediatrics (AAP) in 1998. These included children less than two years of age who had required treatment for CLD within 6 months of the onset of RSV season; infants born at 28 weeks or less gestation without CLD who were less than 12 months of age; infants born at 29-32 weeks gestation who were up to 6 months of age; infants born at 32 to 35 weeks gestation who had additional risk factors such as exposure to tobacco smoke in the home, multiple siblings, attendance at day care centers, or living a long distance from a hospital; and children with severe immunodeficiencies. A written order was obtained from a physician for prophylaxis with palivizumab for each eligible patient.

Once the patient's eligibility and the physician's order was received, in-home RSV prophylaxis with palivizumab was provided by local branches of the home health provider. A nurse contacted the patient's family to arrange for each monthly home visit. Vials of palivizumab and related supplies were shipped to the family's home for arrival one day before each scheduled visit.

\section{Interventions and Outcome Measurement}

At each home visit, which lasted approximately one hour, thenurse assessed the infant or child, reconstituted the dose over the required 30 minutes, conducted a parent education session, administered the appropriate dose of palivizumab, and interviewed one or both parents to collect baseline and monthly outcome data. The parent education session included information about RSV, palivizumab, child-care, and child growth and development. In addition, the nurse observed the patient for at least 30 minutes after the palivizumab injection for any adverse reactions.

The parent interview provided outcome data, which the nurse documented on a visit log at the time of the visit. Indicators of program effectiveness included incidence of RSVrelated hospitalization and LOS, LOS in the ICU, ED visits, and incidence and types of adverse reactions. Parents were asked to report any illnesses the infant or child had experienced since 


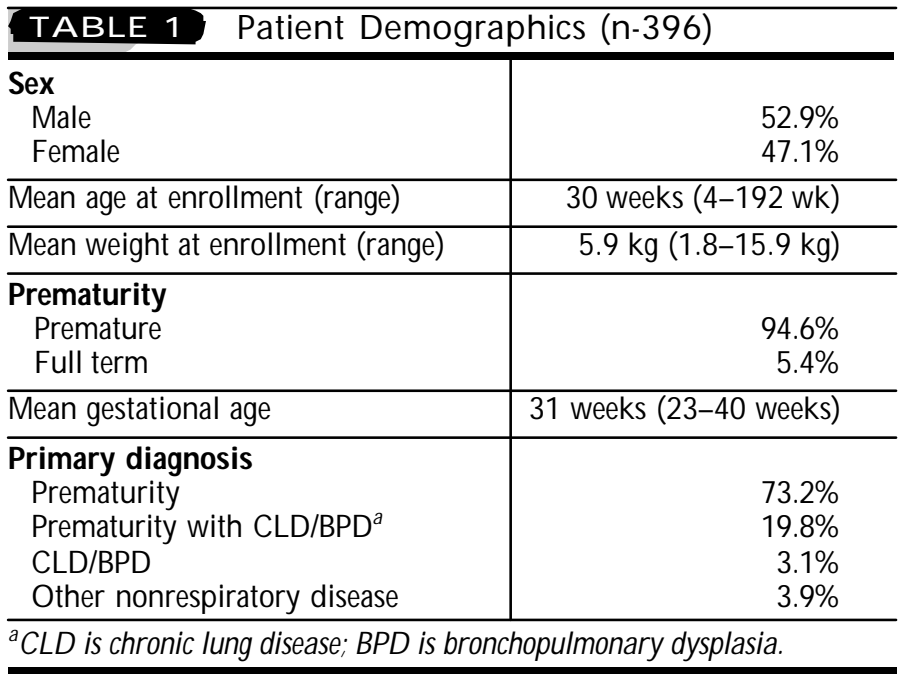

the last visit, any hospitalizations or ED visits needed since the previous visit, and whether RSV was the principal diagnosis for the hospitalization or ED visit. At the last home visit, the nurse also asked the parents to complete a satisfaction survey. The written survey was given to the parents with a stamped return envelope, and the parents mailed back the completed survey.

\section{Analysis}

Surveys were compiled by EPI-Q Inc. (Oakbrook Terrace, IIIinois), an independent outcomes measurement organization that entered the data into a computerized database. Descriptive analy- ses were performed to quantify patient demographics, treatment practice, and parent satisfaction with the program. Cost parameters for administering the in-home program were provided from internal Gentiva cost-accounting data. The literature was reviewed to obtain clinical benchmarks for incidence of RSV, emergency room visits, hospitalizations, and LOS in non-homecare settings. Results from the pivotal study published by the Impact-RSV Study Group were used as a comparison to the descriptive outcomes obtained. ${ }^{2}$ This benchmark included data on 1,002 patients receiving palivizumab as outpatients.

Categorical incidence data (e.g., hospitalizations, emergency room visits) were compared to published benchmarks using $\left.\right|^{2}$ statistics. Economic benchmarks for cost of clinic-based prophylaxis programs and RSV hospitalization emergency room visits were also abstracted from the published palivizumab cost-effectiveness literature for comparison to program results. ${ }^{6,7}$ Costs were defined as the variable costs associated with the provideradministered care (nursing visit cost, drug acquisition cost, cost of hospitalization).

\section{Results}

Patients

During the 1999-2000 RSV season 396 patients were enrolled and treated in the palivizumab in-home RSV prophylaxis program; all were included in the analysis. The patient population was $52.9 \%$ male and $47.1 \%$ female with a mean age of approximately 30 weeks (range: 4-192 weeks) at enrollment (see Table

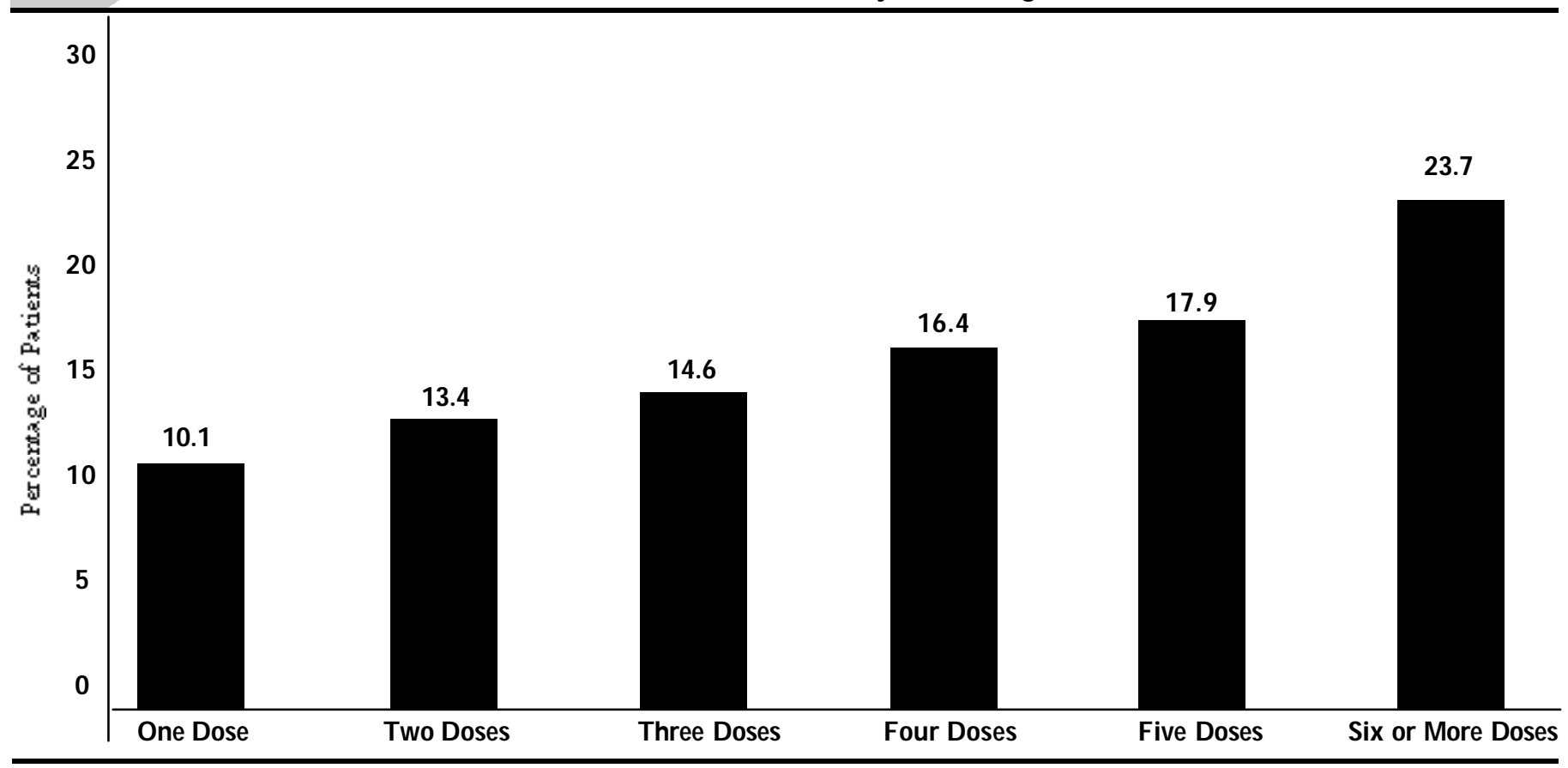




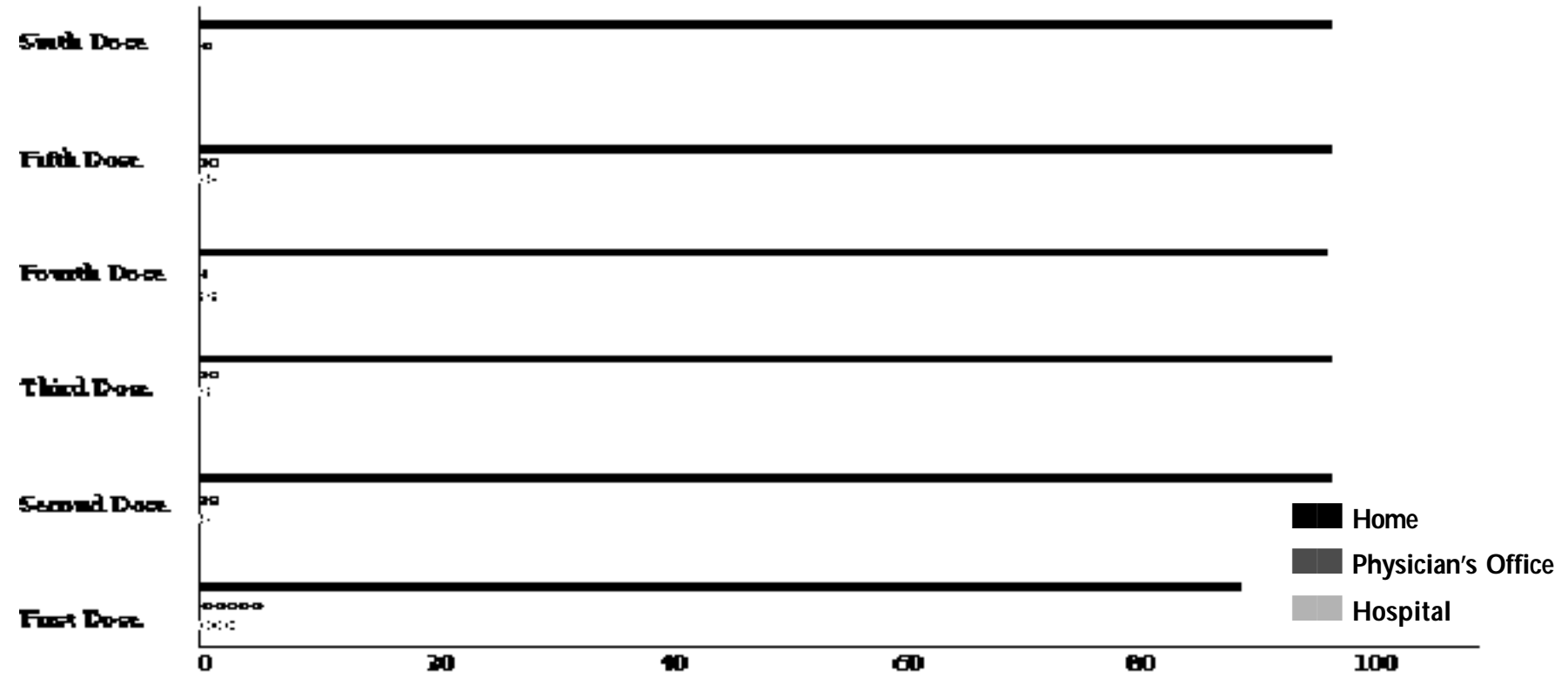

1, page 478). The vast majority of patients were premature at birth; only $5.4 \%$ were full-term infants. The mean gestational age was 31 weeks (range: 23-40 weeks), and the mean birth weight was $1.78 \mathrm{~kg}$ (range: $0.5-6.5 \mathrm{~kg}$ ).

The primary diagnosis indicating the need for RSV prophylaxis was prematurity either alone (73.2\% of patients) or with CLD or BPD (19.8\%). A small proportion of patients (7.0\%) had CLD or BPD or a nonrespiratory illness as the primary indication for RSV prevention.

The number of intramuscular doses of palivizumab administered was available for approximately $96 \%$ of the patients (see Figure 1, page 478). Each received a mean of 3.8 doses. N early $24 \%$ of the infants and children were given six or more doses; $15 \%$ to $18 \%$ received three to five doses. The variation in the number of doses reflects the time during the RSV season when prophylaxis was ordered.

About $90 \%$ of patients received their first dose of palivizumab in the home; about $6 \%$ in a physician's office, and about $2 \%$ at a hospital (see Figure 2, above). The second through sixth doses were administered at home for more than $96 \%$ of the patients.

Outcomes from the patients receiving immunization in the home were compared to a cohort of 1,002 patients who had received the drug in an office or clinic setting. ${ }^{2}$ Patients were comparable in terms of gender $(52.9 \%$ male in the in-home patients versus $56.9 \%$ ), gestational age (31 weeks in the in-home patients versus 29). The in-home treated patients were slightly older at immunization onset ( 30 weeks versus 23 ), but this difference was accounted for by one child in the in-home group who received immunization at 192 weeks (3.7 years). Mean age in the in-home group without this outlier child was identical to the clinic-treated patients at 23 weeks.

\section{Clinical Outcomes}

For in-home RSV prophylaxis with palivizumab, adverse events were reported for only 7 of 396 infants and children (1.8\%); all 7 experienced minor inflammation at the intramuscular injection site, accounting for 11 reports of inflammation in 1,499 doses of palivizumab administered (0.7\%). The data are consistent with published benchmarks documenting inflammation rates at $2.3 \%{ }^{2}$

Parents reported cold symptoms in $43.2 \%$ of the patients, ear infection in $25.3 \%$, and fever in $23.3 \%$ of the patients at some point during treatment. The incidence of total hospitalizations was $11.4 \%$, of which $2.3 \%$ was attributable to RSV (see Figure 3, page 480). Only one of the hospitalized patients required admission to the ICU, and was treated there for a total of four days. The incidence of ED visits was $9.8 \%$, but only $1 \%$ of these visits were for treatment of RSV illness.

Hospitalization was required to treat pneumonia in $2.5 \%$ and other respiratory illness in $4.3 \%$ of the patients; these same conditions prompted ED visits for $3.0 \%$ and $4.3 \%$ of the patients. Nonrespiratory illnesses accounted for hospitalization and in $8.3 \%$ and ED visits in $4.0 \%$ of the patients. Another $1.5 \%$ of the patients visited the ED for ear infections.

Illnesses reported during in-home prophylaxis with palivizumab were fewer than those reported during outpatient or clinicbased administration, particularly ear infections $(25.3 \%$ vs. $42.0 \%, p<0.05) .^{2}$ Total hospitalizations for any reason and hospitalizations related only to RSV were lower during in-home pro- 


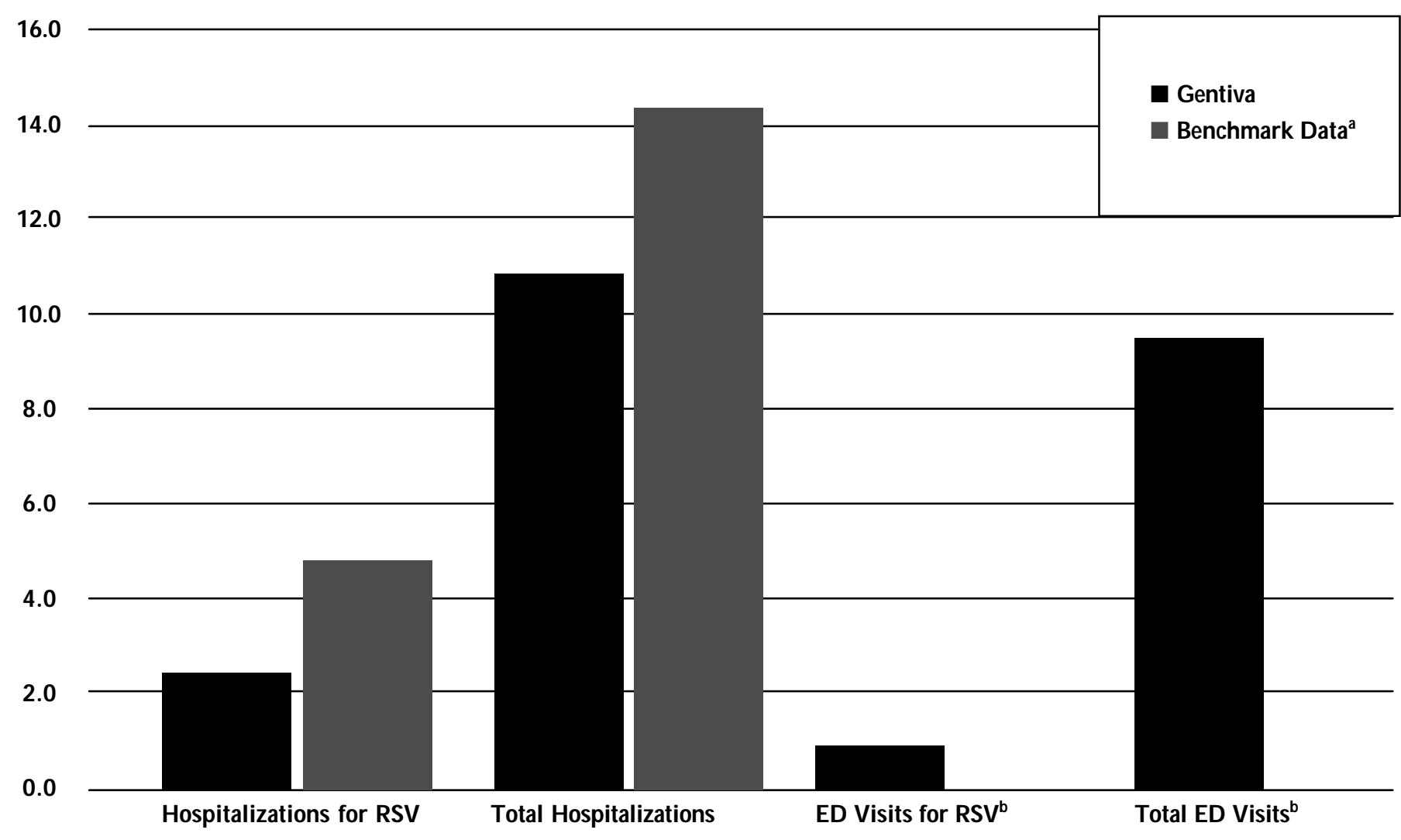

${ }^{a} \mathrm{~N}$ o outpatient clinic benchmark available

benchmark data abstracted from the Impact-RSV Study Group

phylaxis (11.4\% and $2.3 \%)$ than during outpatient $(14.4 \%)$ or clinic administration (4.8\%). ${ }^{2}$ This compares favorably to clinical trials of RSV-IGIV and palivizumab, in which infants who did not receive prophylaxis had a total hospitalization rate of $12.3 \% .^{7}$ The $4.8 \%$ rate for infants who received palivizumab prophylaxis as outpatients represents a $61 \%$ reduction in RSV-related hospitalization. ${ }^{7}$ W ith in-home palivizumab prophylaxis, the reduction in RSV-related hospitalization (2.3\% versus $12.3 \%$ ) was calculated as $81 \%$. The incidences of total ED visits and RSV-related ED visits were low during in-home palivizumab prophylaxis.

\section{Satisfaction Outcomes}

Nearly all parents (98.6\%) who responded to the satisfaction survey were either satisfied or very satisfied with the palivizumab inhome RSV prophylaxis program, and the vast majority (91.0\%) indicated that they would prefer in-home prophylaxis for RSV if they were to have another infant or child at high risk.

In addition to high parental satisfaction with in-home RSV prophylaxis with palivizumab, there was a high degree of compliance. The program was completed by $85 \%$ of the infants and children enrolled, and completion usually coincided with the end of the RSV season. Less than $2 \%$ of the patients discontinued due to noncompliance. Other reasons for discontinuation included relocation of the family, a change in health services agency, or a change in third-party payer requirements.

\section{Cost Outcomes}

The direct cost to the provider of administering palivizumab by a nurse in the home-care setting was calculated at $\$ 65$ per dose. With a mean of 3.8 doses per infant or child, the total drug administration cost was $\$ 247$ (see Table 2, page 481). Based on a cost per hospitalization of $\$ 10,851$ (1994 dollars adjusted for $4 \%$ annual inflation) and a hospitalization rate during the inhome prophylaxis program of 0.023 , total hospitalization costs for each infant immunized were $\$ 250$, for a total in-home program cost of $\$ 497$ for each infant or child. ${ }^{6}$

\section{Conclusions}

The Gentiva program for RSV prophylaxis demonstrated that an in-home program is a viable alternative to clinic-based adminis- 


\begin{tabular}{|c|c|c|}
\hline & $\begin{array}{l}\text { Home } \\
\text { Care }\end{array}$ & $\begin{array}{l}\text { Outpatient/ } \\
\text { Clinic }\end{array}$ \\
\hline Drug administration cost & $\$ 65$ & $\$ 50^{\mathrm{a}}$ \\
\hline $\begin{array}{l}\text { Number of doses } \\
\text { per infant/child }\end{array}$ & 3.8 & 3.8 \\
\hline $\begin{array}{l}\text { Calculated cost of drug } \\
\text { administration per infant/child }\end{array}$ & $\$ 247$ & $\$ 190$ \\
\hline Hospitalization rate & 0.023 & $0.048^{b}$ \\
\hline Cost per hospitalization & $\$ 10,851$ & $\$ 10,851$ \\
\hline $\begin{array}{l}\text { Calculated hospital costs per } \\
\text { infant/child immunized }\end{array}$ & $\$ 250$ & $\$ 521$ \\
\hline $\begin{array}{l}\text { Calculated costs of drug } \\
\text { administration and hospitalization } \\
\text { per infant/child immunized }\end{array}$ & $\$ 497$ & $\$ 711$ \\
\hline
\end{tabular}

${ }^{a}$ Based on costs used in a published cost-effectiveness study. See endnote 6. ${ }^{b} \mathrm{H}$ ospitalization rate in a published report of palivizumab prophylaxis. See endnote 2.

tration. Intramuscular administration of palivizumab at home can improve clinical outcomes compared with published outcomes for outpatient administration. ${ }^{2}$ As a result, the infants and children given palivizumab prophylaxis used fewer resources than published benchmarks for outpatient or clinic care. ${ }^{2}$

The parents of the infants and children who received palivizumab at home were overwhelmingly satisfied with the program. Parental satisfaction, coupled with favorable clinical outcomes, resulted in a high degree of compliance to the six scheduled monthly intramuscular injections of palivizumab. Less than $2 \%$ of the patients discontinued the program due to noncompliance, and more than $90 \%$ of parents indicated they would choose in-home prophylaxis if they had another child at high-risk of RSV illness.

In-home RSV prophylaxis with palivizumab addresses several disadvantages of office-based administration, increasing compliance. Home administration minimizes the exposure of high-risk infants to communicable diseases; it is also more convenient for parents of multiple premature infants and infants who require numerous cumbersome medical devices. In the clinic or physician's office, infants receiving palivizumab are often placed in an examination room while the lyophilized powder is reconstituted; the reconstituted vial must sit at room temperature for 20 minutes. After injection, the infant must be observed for another 30 minutes; thus, the examination room is unavailable for other use for at least 50 minutes. At home, on the other hand, this time provides an opportunity for an expe- rienced pediatric nurse to educate parents.

There is no dispute that prophylaxis against RSV infection, as recommended by the AAP, reduces the morbidity and mortality of RSV-related illness in the pediatric population; however, its high cost is a serious concern. ${ }^{5,6}$ Office-based physicians may be concerned that in using RSV prophylaxis they may incur additional inventory costs and fees for billing services, whereas the health services agency providing in-home RSV prophylaxis can manage its own inventory and provide benefits verification and billing services. With the Gentiva in-home program, the decrease in hospitalization during prophylaxis with palivizumab realized a cost savings of $\$ 250$ per patient treated compared with an outpatient or clinic-based program (Table 2). ${ }^{2,6}$ Although the per-dose cost of administering the drug in the outpatient clinic setting was lower ( $\$ 50$ versus $\$ 65)$, the higher rate of hospitalization would increase the total cost of the prophylaxis program and hospital ization for each infant or child treated ( $\$ 711$ versus $\$ 497$ ).

When drug costs are included, the difference in total costs between in-home administration and physician office or clinic administration are small indeed. Assuming acquisition costs of $\$ 785$ per dose, $\$ 50$ for an office visit, and $\$ 65$ for a home nursing visit, the cost of a series of six injections would be $\$ 5,010$ for prophylaxis with palivizumab in the office setting and $\$ 5,100$ in the home care setting, a difference of less than $2 \%$. In-home administration appears to be cost effective compared with administration in other sites of care. In-home administra tion is also associated with excellent clinical outcomes, very high compliance, and very high parental satisfaction.

\section{References}

1. Robinson RF, Nahata MC. Respiratory syncytial virus (RSV) immune globulin and palivizumab for prevention of RSV infection. Am J Health-Syst Pharm 2000; 57: 259-67.

2. Palivizumab, a humanized respiratory syncytial virus monoclonal antibody, reduces hospitalization from respiratory syncytial virus infection in high-risk infants. The Impact-RSV Study Group. Pediatrics 1998; 102: 531-37.

3. Scott LJ, Lamb HM. Palivizumab. Drugs 1999; 58: 305-11.

4. Saez-Llorens $X$ et al. Safety and pharmacokinetics of an intramuscular humanized monoclonal antibody to respiratory syncytial virus in premature infants and infants with bronchopulmonary dysplasia. Pediatr Infect Dis J 1998; 17: 787-91.

5. Prevention of respiratory syncytial virus infections: indications for the use of palivizumab and update on the use of RSV-IGIV. Pediatrics 1998; 102: 1211-16.

6 . Joffe $S$ et al. Cost-effectiveness of respiratory syncytial virus prophylaxis among preterm infants. Pediatrics 1999; 104: 419-27.

7. Marchetti A et al. Impact of palivizumab on expected costs of respiratory syncytial virus infection in preterm infants: potential for savings. Clin Therap 1999; 21: 752-66. 